\title{
A 3-form gauge potential in 5D in connection with a possible dark sector of 4D-electrodynamics
}

\author{
D. Cocuroci ${ }^{1, \mathrm{a}}$, M. J. Neves ${ }^{2, \mathrm{~b}}$, J. A. Helayël-Neto ${ }^{1, \mathrm{c}}$, L. P. R. Ospedal ${ }^{1, \mathrm{~d}}$ \\ ${ }^{1}$ Centro Brasileiro de Pesquisas Físicas, Rua Dr. Xavier Sigaud 150, Urca, Rio de Janeiro CEP 22290-180, Brazil \\ ${ }^{2}$ Universidade Federal Rural do Rio de Janeiro, BR 465-07, Seropédica, Rio de Janeiro 23890-971, Brazil
}

Received: 31 January 2015 / Accepted: 25 June 2015 / Published online: 11 July 2015

(C) The Author(s) 2015. This article is published with open access at Springerlink.com

\begin{abstract}
We here propose a 5-dimensional Abelian gauge model based on the mixing between a $U(1)$ potential and an Abelian 3-form field by means of a topological mass term. An extended covariant derivative is introduced to minimally couple a Dirac field to the $U(1)$ potential, while this same covariant derivative non-minimally couples the 3 -form field to the charged fermion. A number of properties are discussed in 5D; in particular, the appearance of a topological fermionic current. A 4-dimensional reduced version of the model is investigated and, in addition to the $U(1)$ electricand magnetic-sort of fields, there emerges an extra set of electric- and magnetic-like fields which contribute a negative pressure and may be identified as a possible fraction of dark energy. The role of the topological fermionic current is also contemplated upon dimensional reduction from $5 \mathrm{D}$ to $4 \mathrm{D}$. Other issues we present in 4 space-time dimensions are the emergence of a pseudo-scalar massive particle, an extra massive neutral gauge boson, which we interpret as a kind of paraphoton, and the calculation of spin- and velocitydependent interparticle potentials associated to the exchange of the intermediate bosonic fields of the model.
\end{abstract}

\section{Introduction}

The possibility of a multidimensional Universe has raised a growing interest over the past decades. Currently, the reasons for this interest come primarily from approaches such as superstring theory, which is able to incorporate quantum gravity in a natural and consistent way [1].

As a consequence of the superstring landscape, it is nowadays widely accepted that the structure of space-time must

\footnotetext{
a e-mail: denis.cocuroci@gmail.com; cocuroci@cbpf.br

be-mail: mariojr@ufrrj.br

c e-mail: helayel@cbpf.br

de-mail: leoopr@cbpf.br
}

be described as the product of a 5-dimensional anti-de Sitter space by a 5-dimensional hypersphere. Thus, we adopt the viewpoint that the fundamental physics may be derived from 5 space-time and 5 compact internal dimensions.

In addition, the possibility of an equivalence between a classical gravity theory, defined in a 5-dimensional (spacetime) bulk, and a quantum gauge theory (Yang-Mills) on the corresponding 4-dimensional boundary was first proposed by Maldacena in 1997 [2]. Important aspects of the gravitygauge correspondence were elaborated in articles by Gubser et al., and by Witten [3-5].

We shall not, however, adopt the $A d S_{5} / C F T_{4}$ equivalence in its full sense. What we borrow from this correspondence is simply the point of view that our fundamental physics takes place in 5 space-time dimensions; whether this physics should be specifically analyzed in an $A d S_{5}$ or a 5D Minkowski scenario will actually depend on the particular phenomenon under study. Here, we shall assume that, so long as the energy scale for electromagnetic interactions is considered, we do not need to consider the presence of a cosmological constant in the 5-dimensional world. For the investigation we aim to pursue, our starting point is indeed a 5-dimensional Minkowski space-time.

Actually, in the present study, we explore the consequences of an extra dimension [6], by just considering Minkowski space as the background space-time, because the effect of the curvature of the anti-de Sitter space (induced by a cosmological constant, which for the LAMBDA-CDM model is taken to be $10^{-47} \mathrm{GeV}^{4}$ [7]) yields negligible corrections as compared to the scale of masses and lengths typical of QED processes [8]. By neglecting the cosmological constant, the isometry group of $A d S_{5}$ (namely, $S O(2,4)$ ) reduces to the Poincaré group in 5 dimensions. So, we shall here consider a model for electromagnetic interactions in a 5-dimensional Minkowski space and our 4-dimensional physics must emerge as the result of a specific dimensional reduction scheme rather than by holographic projection. 
It is noteworthy that, if we were considering the quantum effects of gravitation, the cosmological constant should not be neglected, for it is well known that the latter induces the production of gravitons with mass of the order of the Planck mass $[9,10]$. However, in the particular case we are concerned to study, massive gravitons do not couple to the associated fluctuations of the electron and photon due to the fact that they are highly massive, so that, in the energy regime of the validity of the QED processes, those gravitons with so a huge mass (induced by the cosmological constant) are not excited.

From this perspective, in this paper, we start off from a model based on the association of a 3 -form gauge potential with accelerated expansion of the Universe [11]. The introduction of the concept of dark energy is actually one of the main approaches to account for the phenomenon of a Universe in accelerated expansion [12]. Our particular model, formulated in 5 space-time dimensions, as already anticipated above, also yields, upon a dimensional reduction mechanism, the appearance of an extra neutral massive boson in 4-dimensional Minkowski space [13-15]. This shall be presented in detail in the sequel. We are actually interested in a gauge-invariant mass term which plays the role of a mixed Chern-Simons topological mass, as it may provide a scenario in 4 space-time dimensions where an axionic particle and a sort of paraphoton emerge together. The pseudo-scalar (axion) and the pseudo-vector are unified in the 5-dimensional world through a topological mass term.

In connection with the study of the 3 -form potential $[11,16-31]$, the mass of the photon is included in order to seek a situation that is as broad as possible, i.e., capable of exploring all the possibilities that a 3-form may offer. According to the work by Koivisto and Nunes [11], 3-forms are used to describe the dark energy fraction of our presently expanding Universe. We should, however, point out that the 3-form potential was initially studied by these authors through a kinetic term (minimally coupled to Einstein gravity) added up to a potential term [28]. Subsequently, the 3-form was reassessed to include coupling to point particles [30]. Here, we intend to investigate the 3-form in association with an Abelian gauge vector, in a 5D scenario, by introducing a topological (mixed) Chern-Simons-like mass term.

On the other hand, in a recent paper [32], the authors show how a vortex gauge field, whenever coupled to charged fermions, induce, by radiative corrections, a gauge-invariant mass term for the photon. Rather than as a dynamical effect, like in the paper [32], in our work, this mass term arises from a dimensional reduction from the $5 \mathrm{D}$ model where there is a topological mass term, as it is going to be shown in the subsequent sections.

Five-dimensional Chern-Simons theory in its Abelian version has recently been studied by Qi, Witten and Zhang
(QWZ) in the context of the physics of topological superconductors [33]. We also take this remarkable contribution-in addition to the $A d S_{5} / C F T_{4}$ correspondence-as a motivation for our exploitations in a 5-dimensional space-time. As is well known, in superconductivity, a massive photon must be present to accommodate the Meissner effect, responsible for the expulsion of the magnetic field from inside materials in their superconducting phase. Thus, with the physics of topological superconductors being processed in 5 dimensions, according to the QWZ scenario, the photon could acquire mass through a mechanism of topological mass generation, as we are going to present here.

In summary, we intend to explore an electrodynamic model that uses both a 3 -form and a 1 -form gauge potentials in a mixed way in order to generate a massive gauge boson in a 5-dimensional scenario. Upon dimensional reduction [34], we actually attain a model that presents in its spectrum a massive neutral vector boson degenerate (i.e., with the same mass) with a neutral scalar excitation, the latter produced by the mixing between a genuine pseudo-scalar field and a longitudinal vector field. Our work follows the outline below.

In Sect. 2, we present the model we adopt to pursue our investigation. We split it in two subsections, in which we obtain the equation fields, discuss the conservation laws and carry out the dimensional reduction of the model to 4D. Next, in Sect. 3, we add up $U$ (1)-charged fermions to the action of the model in 5D discussed in the previous section. We obtain the fermionic conserved currents in 5D and connect them to the pseudo-tensor current of the paper [32]. Coupling the model to gravity in 5D is also carried out. The 5-dimensional action is then reduced to $4 \mathrm{D}$, and we calculate the propagators of the bosonic sector to read off the spectrum of excitations. As an application, interparticle potentials generated by the intermediation of the bosonic fields exchanged by external currents are worked out. Finally, our Concluding Comments are cast in Sect. 4.

\section{Description of the model}

Taking for granted the importance of understanding physics in our 4-dimensional world from a more fundamental 5dimensional physics, we focus here on a study of a specific electrodynamic model in 5 dimensions aiming at the possible consequences it yields in a 4-dimensional space-time.

Thus, in this section, we present the model which consists of a Lagrangian density containing the kinetic terms for each gauge field $\left(A_{\bar{\mu}}\right.$, and $\left.C_{\bar{\mu} \bar{\nu} \bar{\kappa}}\right)$, and a mixing term between them. This mixing term is capable of ensuring that the mass of the associated particle is independent of the metric characteristics of the space. It is known in the literature as a topological term [35-37]. We also exhibit the field equations, the Bianchi identities and the conservation laws. 
Consider the action in 5D whose corresponding Lagrangian density is as follows:

$$
\mathcal{L}=-\frac{1}{4} F_{\bar{\mu} \bar{\nu}} F^{\bar{\mu} \bar{\nu}}+\alpha H_{\bar{\mu} \bar{\nu} \bar{\kappa} \bar{\lambda}} H^{\bar{\mu} \bar{\nu} \bar{\kappa} \bar{\lambda}}+\beta \epsilon^{\bar{\mu} \bar{\nu} \bar{\kappa} \bar{\lambda} \bar{\rho}} A_{\bar{\mu}} \partial_{\bar{\nu}} C_{\bar{\kappa} \bar{\lambda} \bar{\rho}},
$$

where $A_{\bar{\mu}}$ is the Abelian vector and $C_{\bar{\mu} \bar{\nu} \bar{\kappa}}$ is the 3-form gauge field, one of the main elements of this study. The notation of the indices in 5 dimensions is $\bar{\mu}=\{0,1,2,3,4\}$. The tensor $F_{\bar{\mu}} \bar{\nu}$ is the usual electromagnetic field strength, and the tensor $H_{\bar{\mu} \bar{\nu} \bar{\kappa} \bar{\lambda}}$ is the completely antisymmetric field strength associated with the 3 -form field, $C_{\bar{\mu} \bar{\nu} \bar{\kappa}}$ :

$$
H_{\bar{\mu} \bar{\nu} \bar{\kappa} \bar{\lambda}}=\partial_{\bar{\mu}} C_{\bar{\nu} \bar{\kappa} \bar{\lambda}}-\partial_{\bar{\nu}} C_{\bar{\lambda} \bar{\kappa} \bar{\mu}}+\partial_{\bar{\kappa}} C_{\bar{\lambda} \bar{\mu} \bar{\nu}}-\partial_{\bar{\lambda}} C_{\bar{\mu} \bar{\nu} \bar{\kappa}}
$$

The parameters $\alpha$ and $\beta$ are both real. It is not difficult to check that the $\beta$-parameter has mass dimension. The action defined through the Lagrangian (1) is invariant under the following Abelian gauge transformations in 5D:

$A_{\bar{\mu}} \longmapsto A_{\bar{\mu}}^{\prime}=A_{\bar{\mu}}+\partial_{\bar{\mu}} \Lambda$,

$C_{\bar{\mu} \bar{\nu} \bar{\kappa}} \longmapsto C_{\bar{\mu} \bar{\nu} \bar{\kappa}}^{\prime}=C_{\bar{\mu} \bar{\nu} \bar{\kappa}}+\partial_{\bar{\mu}} \xi_{\bar{\nu} \bar{\kappa}}+\partial_{\nu} \xi_{\bar{\kappa} \bar{\mu}}+\partial_{\bar{\kappa}} \xi_{\bar{\mu} \bar{\nu}}$,

where $\Lambda$ and $\xi_{\bar{\mu} \bar{\nu}}$ are real functions and $\xi_{\bar{\mu} \bar{\nu}}$ is an antisymmetric tensor field. The transformation (3) is the one already known from electrodynamics, $U(1)_{A_{\bar{\mu}}}$, whereas (4) is the antisymmetrized version of the gauge transformation for a rank-3 tensor, $U(1)_{C_{\bar{\mu} \bar{\nu} \bar{\kappa}}}$. Thus, the action is said to be $U(1)_{A_{\bar{\mu}}} \otimes U(1)_{C_{\bar{\mu} \bar{\nu} \bar{\kappa}}}$-invariant. The Lagrangian (1) gives us the field equations

$\partial_{\bar{\mu}} F^{\bar{\mu} \bar{\nu}}+6 \beta \tilde{H}^{\bar{\nu}}=0$,

$8 \alpha \partial_{\bar{\mu}} H^{\bar{\mu} \bar{\nu} \bar{\kappa} \bar{\lambda}}-\beta \widetilde{F}^{\bar{\nu} \bar{\kappa} \bar{\lambda}}=0$.

where the relations between the dual tensors $\widetilde{F}^{\bar{\mu}} \bar{\nu} \bar{\kappa}$ and $F_{\bar{\mu} \bar{\nu}}$ are given by the expressions

$F_{\bar{\mu} \bar{\nu}}=-\frac{1}{3 !} \epsilon_{\bar{\mu} \bar{\nu} \bar{\kappa} \bar{\alpha} \bar{\beta}} \widetilde{F}^{\bar{\mu} \bar{\nu} \bar{\kappa}} \quad$ and $\quad \widetilde{F}_{\bar{\mu} \bar{\nu} \bar{\kappa}}=\frac{1}{2 !} \epsilon_{\bar{\mu} \bar{\nu} \bar{\kappa} \bar{\lambda} \bar{\rho}} F^{\bar{\lambda} \bar{\rho}}$.

As for the relations between $\widetilde{H}^{\bar{\mu}}$ and $H_{\bar{\mu} \bar{\nu} \bar{\kappa} \bar{\lambda}}$, the expressions are given by

$H_{\bar{\mu} \bar{\nu} \bar{\kappa} \bar{\lambda}}=\epsilon_{\bar{\mu} \bar{\nu} \bar{\kappa} \bar{\lambda} \bar{\rho}} \widetilde{H}^{\bar{\rho}} \quad$ and $\quad \widetilde{H}_{\bar{\mu}}=\frac{1}{4 !} \epsilon_{\bar{\mu} \bar{\nu} \bar{\kappa} \bar{\lambda} \bar{\rho}} H^{\bar{\nu} \bar{\kappa} \bar{\lambda} \bar{\rho}}$.

The Bianchi identities associated to the fields $F^{\bar{\mu} \bar{\nu}}$ and $H_{\bar{\mu} \bar{\nu} \bar{\kappa} \bar{\lambda}}$ are, respectively:

$$
\begin{aligned}
& \partial_{\bar{\mu}} F_{\bar{\nu} \bar{\kappa}}+\partial_{\bar{\nu}} F_{\bar{\mu} \bar{\kappa}}+\partial_{\bar{\kappa}} F_{\bar{\mu} \bar{\nu}}=0, \\
& \partial_{\bar{\mu}} H_{\bar{\nu} \bar{\kappa} \bar{\lambda} \bar{\rho}}+\partial_{\bar{\nu}} H_{\bar{\kappa} \bar{\lambda} \bar{\rho} \bar{\mu}}+\partial_{\bar{\kappa}} H_{\bar{\nu} \bar{\lambda} \bar{\mu} \bar{\rho}} \\
& \quad+\partial_{\bar{\lambda}} H_{\bar{\nu} \bar{\kappa} \bar{\rho} \bar{\mu}}+\partial_{\bar{\rho}} H_{\bar{\mu} \nu \bar{\kappa} \bar{\lambda}}=0 .
\end{aligned}
$$

Expression (10) can also be cast in a more compact form in terms of the dual of $H_{\bar{\mu} \bar{\nu} \bar{\kappa} \bar{\lambda}}$, i.e.:

$\partial_{\bar{\mu}} \widetilde{H}^{\bar{\mu}}=0$.
The field equations (5) and (6) are coupled and we must necessarily decouple them in order to implement the procedure that will reveal the mass of the particle(s) associated(s) with both fields. It yields

$$
\left(\square-\frac{3}{4 \alpha} \beta^{2}\right) F_{\bar{\mu} \bar{\nu}}=0,
$$

and

$$
\left(\square-\frac{3}{4 \alpha} \beta^{2}\right) H_{\bar{\mu} \bar{\nu} \bar{\kappa} \bar{\lambda}}=0 .
$$

Therefore, it is noted from (12) and (13) that both fields are shown to exhibit the same mass term, which is given by $\xi=\frac{-3 \beta^{2}}{4 \alpha}$, where it is considered that the parameter $\alpha$ must be restricted to a negative real number. The energy-momentum tensor is obtained by multiplying Eq. (5) by $F_{\bar{\nu}} \bar{\alpha}$ and using the following relation between the dual fields:

$\widetilde{F}^{\bar{\nu} \bar{\kappa} \bar{\lambda}} H_{\bar{\nu} \bar{\kappa} \bar{\lambda} \bar{\alpha}}=-6 F_{\bar{\nu} \bar{\alpha}} \widetilde{H}^{\bar{\nu}}$

Then we insert (6) to get

$$
\begin{aligned}
& -\partial_{\bar{\mu}}\left(8 \bar{\alpha} H^{\bar{\mu} \bar{\nu} \bar{\kappa} \bar{\lambda}} H_{\bar{\nu} \bar{\kappa} \bar{\lambda} \bar{\alpha}}\right)+8 \alpha H^{\bar{\mu} \bar{\nu} \bar{\kappa} \bar{\lambda}} \partial_{\bar{\mu}} H_{\bar{\nu} \bar{\kappa} \bar{\lambda} \bar{\alpha}} \\
& +\partial_{\bar{\mu}}\left(F^{\bar{\mu} \bar{\nu}} F_{\bar{\nu} \bar{\alpha}}\right)-F^{\bar{\mu} \bar{\nu}} \partial_{\bar{\mu}} F_{\bar{\nu} \bar{\alpha}}=0 .
\end{aligned}
$$

Thus, replacing the Bianchi identities (9), (10), and using the relation

$2 F_{\bar{\nu} \bar{\alpha}} \partial_{\bar{\mu}} F^{\bar{\mu} \bar{\nu}}=\partial_{\bar{\mu}}\left(16 \alpha H^{\bar{\mu} \bar{\nu} \bar{\kappa} \bar{\lambda}} H_{\bar{\nu} \bar{\kappa} \bar{\lambda} \bar{\alpha}}\right)+\partial_{\bar{\alpha}}\left(2 \alpha H_{\bar{\mu} \bar{\nu} \bar{\kappa} \bar{\lambda}}^{2}\right)$,

we obtain the continuity equation

$\partial_{\bar{\mu}} \Theta_{\bar{\alpha}}^{\bar{\mu}}=0$,

where $\Theta_{\bar{\alpha}}^{\bar{\mu}}$, the energy-momentum tensor associated with the Lagrangian (1), is given by

$$
\begin{aligned}
\Theta_{\bar{\alpha}}^{\bar{\mu}}= & -8 \alpha\left(H^{\bar{\mu} \bar{\nu} \bar{\kappa} \bar{\lambda}} H_{\bar{\nu} \bar{\kappa} \bar{\lambda} \bar{\alpha}}+\delta^{\bar{\mu}} \frac{1}{8} H_{\bar{\mu} \bar{\nu} \bar{\kappa} \bar{\lambda}}^{2}\right) \\
& +F^{\bar{\mu} \bar{\nu}} F_{\bar{\nu} \bar{\alpha}}+\delta_{\bar{\alpha}}^{\bar{\mu}} \frac{1}{4} F_{\bar{\mu} \bar{\nu}}^{2} .
\end{aligned}
$$

Comparing the second term of $\Theta_{\bar{\alpha}}^{\bar{\mu}}$ with the kinetic term of the rank-3 tensor field in (1), we can set the value of the parameter as $\alpha=-1 / 8$. Thus, we rewrite the mass as $m^{2}:=\frac{-3 \beta^{2}}{4 \alpha}$ and thus the value of $\beta$ is fixed. Therefore, the topological mass term, $\Delta$, is given by

$\Delta=\frac{m}{\sqrt{6}} \epsilon^{\bar{\mu} \bar{\nu} \bar{\kappa} \bar{\lambda} \bar{\rho}} A_{\bar{\mu}} \partial_{\bar{\nu}} C_{\bar{\kappa} \bar{\lambda} \bar{\rho}}$.

The energy-momentum tensor is written in terms of the fieldstrength tensors, then it is naturally invariant under the gauge transformations (3) and (4). It is also symmetrical; the expression (18) can be rewritten in terms of the dual field of $H_{\bar{\mu} \bar{\nu} \bar{\kappa} \bar{\lambda}}$ as follows:

$\Theta_{\bar{\alpha}}^{\bar{\mu}}=6 \widetilde{H}^{\bar{\mu}} \widetilde{H}_{\bar{\alpha}}-\delta_{\bar{\alpha}}^{\bar{\mu}} 3 \widetilde{H}_{\bar{\mu}}^{2}+F^{\bar{\mu} \bar{\nu}} F_{\bar{\nu} \bar{\alpha}}+\delta^{\bar{\mu}}{ }_{\bar{\alpha}} \frac{1}{4} F_{\bar{\mu} \bar{\nu}}^{2}$. 
Table 1 Components of tensor field $F^{\bar{\mu} \bar{\nu}}$ and dual tensor of $H^{\bar{\mu} \bar{\nu} \bar{\rho} \bar{\lambda}}$. The indices $i, j, k=1,2,3$ refer to the space components in 4-dimensional space-time

\begin{tabular}{ll}
\hline$F^{\bar{\mu} \bar{\nu}}$ & $\widetilde{H}^{\bar{\mu}}$ \\
\hline$F^{0 i}=-\vec{E}_{i}$ & $\widetilde{H}^{0}=\chi$ \\
$F_{i j}=-\epsilon_{i j k} \overrightarrow{B_{k}}$ & $\widetilde{H}^{i}=\vec{Y}_{i}$ \\
$F^{04}=-b$ & $\widetilde{H}^{4}=S$ \\
$F^{i 4}=\overrightarrow{e_{i}}$ & \\
\hline
\end{tabular}

2.1 Decomposition into irreducible components of $S O(3)$

To carry out the decomposition of the energy-momentum tensor (20), the field equations (5), (6), and the Bianchi identities (9) and (11) in terms of irreducible components of $S O(3)$, we initially make the identification of each sector of $F^{\mu \nu}$ and $\widetilde{H}^{\mu}$ with the corresponding irreducible components of $S O(3)$ as listed in Table 1:

From (17), we extract the components of the conserved energy-momentum tensor $\Theta_{\bar{\alpha}}^{0}$, so that the energy, the Poynting vector, and a new density pressure associated with the extra dimension are expressed, respectively, as follows:

$$
\begin{aligned}
\Theta_{0}^{0} & =\frac{1}{2}\left(E^{2}+B^{2}+b^{2}+e^{2}\right)-3\left(\chi^{2}+Y^{2}+S^{2}\right) \\
\Theta_{i}^{0} & =-(\vec{E} \times \vec{B})_{i}+b \overrightarrow{e_{i}}+6 \chi \overrightarrow{Y_{i}} \\
\Theta^{0}{ }_{4} & =-\vec{E} \cdot \vec{e}+6 \chi S .
\end{aligned}
$$

Going on with the procedure for extracting the components of the energy-momentum tensor, we see that the stress tensors read

$$
\begin{aligned}
\Theta_{i j} & =-\vec{E}_{i} \overrightarrow{E_{j}}-\overrightarrow{B_{i}} \overrightarrow{B_{j}}+\overrightarrow{e_{i}} \overrightarrow{e_{j}}-6 \overrightarrow{Y_{i}} \vec{Y}_{j} \\
\Theta_{i 4} & =(b \vec{E}+\vec{e} \times \vec{B})_{i}-6 \overrightarrow{Y_{i}} S \\
\Theta_{44} & =\frac{1}{2}\left(E^{2}-B^{2}+e^{2}-b^{2}\right)-3\left(S^{2}+\chi^{2}-Y^{2}\right) .
\end{aligned}
$$

In Table $1, \vec{E}, \vec{B}, \chi$ and $\vec{Y}$ are the field strengths associated to the Maxwell-like field and the 2-form potential, respectively. On the other hand, $\vec{e}, b$ and $S$ constitute what we call the dark sector of our extended 4-dimensional electrodynamics [13]. We name it dark sector because it is connected to the 3 -form potential whose gauge symmetry is not associated to any sort of matter charge, contrary to the $U(1)$-symmetry of vector bosons whose corresponding charge appears in phase symmetry transformations. At this point, we would like to point out the work of Ref. [38], where the author introduces a second photon, which he refers to as the shadow photon or paraphoton, an unobserved photon. In our case, what we dub as the dark sector is the particle associated to the propagation of $\vec{e}$ and $b$. In our model, there remains a scalar, $S$, which is also part of what we call the dark sector. It would be interesting, but we are not doing this here, if we later work out astrophysical constraints on this dark sector as, it is done in the series of papers quoted in Refs. [39-41].

The right-hand side of Einstein's equation is essentially described by the energy-momentum tensor. This constitutes a unified relation (arising from the space-time symmetry) between the energy density and the pressure in the system. In a 5-dimensional model, one identifies in the energymomentum tensor the presence of a sector able to submit the system, through a particular configuration of the fields (26), to a negative pressure which, in its turn, characterizes the effect of accelerated inflation of the Universe, the effect of the so-called dark energy. As a result of the observations, the inflationary profile of the Universe changes over time $[42,43]$. Currently, it presents itself as accelerated $[44,45]$. This changing behavior in the inflationary profile may be the result of changes in the configuration of the present fields in each phase of the history of the Universe.

In the paper of Ref. [11], the author argues that the tiny value of the cosmological constant can be phenomenologically explained by the use of a 3-form. We also adopt the 3-form, but we consider that, for the sake of electromagnetic effects, the cosmological constant is tiny enough, so that we neglect the curvature of the (anti-de Sitter) space. In view of that, we adopt Minkowski space as the space-time background. Then we attribute to the presence of a specific sector of the energy-momentum tensor in 5D, the effect that mimics dark energy, by virtue of the use of a 3-form in our model.

From what we have discussed above, our work sets out as a possible theoretical support to the paper [11] in order to provide a justification to the fact that the 3 -form potential yields a negative pressure, as suggested by the presence of the $\Theta^{4}{ }_{4}$ component of the energy-momentum tensor (26), which may become negative depending on the particular configuration of the fields ( $\vec{E}, \vec{B}, \vec{e}, b, \chi, S$ and $\vec{Y}$ ).

The topological mass term (19) used in our action does not affect-by construction-the energy-momentum tensor (20), once it is metric-independent. Hence, if the $\Theta_{4}^{4}$ component shows up as a negative contribution, it happens regardless the mass-like term we adopt. This $\Theta_{4}^{4}$, which is negative in $5 \mathrm{D}$, may play the role of the negative pressure associated to a (positive) cosmological constant in 4D, which is a possible landscape to support an accelerated expansion of our Universe.

\subsection{Radiation fields in 4D}

Next we exhibit the field equations in 5D extracted from the Lagrangian (1) where it is considered fixed constants $\alpha$ and $\beta$ as has been detailed in the previous section. The equations are expressed in terms of the components $\vec{E}, \vec{B}, \vec{e}$ and $b$ of 
Table 2 Field equations and their sources

\begin{tabular}{ll}
\hline$\partial_{\mu} H^{\bar{\mu} \bar{\nu} \bar{\kappa}}+\frac{m}{\sqrt{6}} \widetilde{F}^{\bar{\nu} \bar{\kappa} \bar{\lambda}}=J^{\bar{\nu} \bar{\kappa} \bar{\lambda}}$ & $J^{\bar{\nu} \bar{\kappa} \bar{\lambda}}$ \\
\hline$\vec{\nabla} S+\frac{m}{\sqrt{6}}(\vec{e})=\vec{\lambda}$ & $\lambda_{k}=\frac{1}{2} \epsilon_{i j k} J^{0 i j}$ \\
$\vec{\nabla} \times \vec{Y}+\frac{m}{\sqrt{6}} \vec{B}=\vec{\zeta}$ & $\zeta^{i}=J^{0 i 4}$ \\
$\frac{\partial \vec{Y}}{\partial t}+\vec{\nabla} \chi+\frac{m}{\sqrt{6}} \vec{E}=\vec{\sigma}$ & $\sigma_{i}=\frac{1}{2} \epsilon_{i j k} J^{j k 4}$ \\
$\frac{\partial S}{\partial t}+\frac{m}{\sqrt{6}} b=\tau$ & $\tau=-\epsilon_{i j k} J^{i j k}$ \\
\hline
\end{tabular}

$F^{\mu \nu}$ and of the components $\chi, S$ and $\vec{Y}$ of $H_{\mu \nu \kappa \lambda}$ including the mass terms.

We will adopt a dimensional reduction scheme known as Scherk-Schwarz reduction [34] where it is considered that all potentials and fields do not depend on the extra dimension, i.e., it is considered that the derivatives of any field to the fifth coordinate is null, i.e., $\partial_{4}$ (any field) $=0$. The Eq. (5) in the presence of an external source $J^{\mu}$, when it is decomposed reveals the following equations:

$\vec{\nabla} \cdot \vec{E}+m \sqrt{6} \chi=\rho$

$\vec{\nabla} \times \vec{B}+m \sqrt{6} \vec{Y}=\vec{j}+\frac{\partial \vec{E}}{\partial t}$

$\vec{\nabla} \cdot \vec{e}+m \sqrt{6} S=j_{s}+\frac{\partial b}{\partial t}$.

When Eq. (6) is decomposed, it reveals the equations listed on Table 2:

As for the Bianchi identity (9), when it is decomposed reveals:

$\vec{\nabla} \times \vec{E}=-\frac{\partial \vec{B}}{\partial t}$,

$\vec{\nabla} \cdot \vec{B}=0$,

$\vec{\nabla} \times \vec{e}=0$,

$\vec{\nabla} b=\frac{\partial \vec{e}}{\partial t}$.

And finally, the second Bianchi identity gives us just one expression:

$\frac{\partial \chi}{\partial t}+\vec{\nabla} \cdot \vec{Y}=0$.

This is a continuity equation involving the components $(\chi, \vec{Y})$. It appoints that

$\Xi:=\int_{\mathcal{R}} \mathrm{d}^{3} \mathbf{x} \chi(\mathbf{x}, t)$

is a conserved quantity of model.

It is important to clarify that, although we write down and study Maxwell's equations in the 5 dimensions, we shall actually carry out a dimensional reduction to $(1+3) \mathrm{D}$ and, whenever we consider our electromagnetic fields confined to the 4-dimensional space, there appear extra fields which are inherited from 5 dimensions upon our dimensional reduction. So, we are truly considering our electromagnetic interaction in $(1+3) \mathrm{D}$, but we take into account new fields that show up as a by-product of the 5-dimensional space-time where we have set up our physical scenario.

\section{The fermion sector in 5D and its dimensional reduction to $4 \mathrm{D}$}

In this section, we add to the action corresponding to (1) a fermion sector in 5 dimensions:

$$
\begin{aligned}
S_{5 D}= & \int \mathrm{d}^{5} x\left[\bar{\psi}\left(i \gamma^{\bar{\mu}} D_{\bar{\mu}}-m_{f}\right) \psi-\frac{1}{4} F_{\bar{\mu} \bar{\nu}} F^{\bar{\mu} \bar{\nu}}\right. \\
& \left.-\frac{1}{8} H_{\bar{\mu} \bar{\nu} \bar{\kappa} \bar{\lambda}} H^{\bar{\mu} \bar{\nu} \bar{\kappa} \bar{\lambda}}+\frac{m}{\sqrt{6}} \epsilon^{\bar{\mu} \bar{\nu} \bar{\kappa} \bar{\rho} \bar{\rho}} A_{\bar{\mu}} \partial_{\bar{\nu}} C_{\bar{\kappa} \bar{\lambda} \bar{\rho}}\right],
\end{aligned}
$$

where we insert the covariant derivative in order to study the interaction of the Dirac field with the gauge fields

$D_{\bar{\mu}}:=\partial_{\bar{\mu}}+i e A_{\bar{\mu}}+i g \widetilde{H}_{\bar{\mu}}$,

and the spinor $\psi$ is a Dirac fermionic field in 5D. The $\gamma$ matrices are defined as $\gamma^{\bar{\mu}}=\left(\gamma^{\mu}, \gamma^{4}\right)$, with $\gamma^{4}=i \gamma_{5}$ and $\gamma_{5}=i \gamma^{0} \gamma^{1} \gamma^{2} \gamma^{3}$ such that they satisfy the anti-commutation relations

$\left\{\gamma^{\mu}, \gamma^{\nu}\right\}=2 \eta^{\mu \nu}, \quad\left\{\gamma^{\mu}, \gamma_{5}\right\}=0$,

and the conditions $\left(\gamma_{5}\right)^{\dagger}=\gamma_{5}$, and $\left(\gamma_{5}\right)^{2}=1$.

As already stated previously, the fermionic matter is charged only under the $U(1)$-symmetry of the vector field. It has no charge under the Abelian symmetry of the 3-form gauge potential; this is why the latter is only non-minimally coupled to the 3-form $C_{\mu \nu \kappa}$-field.

The field equations derived from for the gauge fields in the presence of fermions are given by

$\partial_{\bar{\mu}} F^{\bar{\mu} \bar{\nu}}+\sqrt{6} m \widetilde{H}^{\bar{\nu}}=e \bar{\psi} \gamma^{\bar{\nu}} \psi$

and

$\partial_{\bar{\mu}} H^{\bar{\mu} \bar{\nu} \bar{\kappa} \bar{\lambda}}+\frac{m}{\sqrt{6}} \widetilde{F}^{\bar{\nu} \bar{\kappa} \bar{\lambda}}=4 g \epsilon^{\bar{\mu} \bar{\rho} \bar{\nu} \bar{\kappa} \bar{\lambda}} \partial_{\bar{\mu}}\left(\bar{\psi} \gamma_{\bar{\rho}} \psi\right)$,

from which we identify the source terms for each equation:

$J_{F}^{\bar{\mu}}=e \bar{\psi} \gamma^{\bar{\mu}} \psi$

and

$J_{H}^{\bar{\mu} \bar{\nu} \bar{\kappa}}=4 g \epsilon^{\bar{\mu} \bar{\nu} \bar{\kappa} \bar{\lambda} \bar{\rho}} \partial_{\bar{\lambda}}\left(\bar{\psi} \gamma_{\bar{\rho}} \psi\right)$.

We may notice that these currents arise due to the presence of the mixing term between the gauge fields in the Lagrangian. $J_{H}^{\bar{\mu} \overline{\bar{\nu}} \bar{\kappa}}$ is a topological current, which means that we have a current that is conserved without any reference to the equations of motion and no continuous symmetry of the Lagrangian 
or the action is associated to this conservation equation. In other words, we have an identically conserved current.

The current $J_{H}^{\bar{\mu} \bar{\nu} \bar{\kappa}}$ above, when dimensionally reduced to $4 \mathrm{D}$, gives rise precisely to the pseudo-tensor current to which the vortex gauge field of [32] couples. In our case, the current stems from the non-minimal coupling present in the covariant derivative (37) as an imprint of the 5-dimensional world. So, this topological current in 5D plays the crucial role of inducing the gauge-invariant mass term of reference [32] upon its coupling to the vortex gauge field.

\subsection{Dimensional reduction}

Next, one redefines the complete action, but now having undergone a procedure of dimensional reduction from 5 to 4 dimensions. The Greek indices follow the notation $\bar{\mu}=(\mu, 4)$ where $\mu$ indicates the usual 4 dimensions and $\bar{\mu}$ indicate 5 dimensions, i.e., the 4 usual dimensions plus an extra spatial dimension.

Here, the 1 -form $A^{\bar{\mu}}$ can be divided into a vector sector and a scalar sector: $A^{\bar{\mu}}=\left(A^{\mu}, A^{4}\right)$. As for the 3 -form, it can be split into two tensor sectors $C^{\bar{\mu} \bar{\nu} \bar{\kappa}}=\left(C^{\mu \nu \kappa}, C^{\mu \nu 4}\right)$. One redefines the scalar component as $A^{4}=\phi$ and one then identifies the sector $C^{\mu \nu 4}=\frac{1}{\sqrt{3}} B^{\mu \nu}$ as the one known in the literature as the Kalb-Ramond field [46].

Thus, the 5D action is reduced to 4D and can be expressed as follows:

$$
\begin{aligned}
S_{4 D}= & \int \mathrm{d}^{4} x\left[\bar{\psi}\left(i \gamma^{\mu} D_{\mu}-m_{f}\right) \psi-\frac{1}{4} F_{\mu \nu}^{2}\right. \\
& +\frac{1}{6} G_{\mu \nu \kappa}^{2}-\frac{2 \sqrt{2}}{3} m \epsilon^{\mu \nu \kappa \lambda} A_{\mu} \partial_{\nu} B_{\kappa \lambda} \\
& +\frac{1}{2}\left(\partial_{\mu} \phi\right)^{2}+\frac{1}{2}\left(\partial_{\mu} X^{\mu}\right)^{2}-m \phi \partial_{\mu} X^{\mu} \\
& \left.+i e \bar{\psi} \gamma_{5} \psi \phi+\frac{i}{\sqrt{6}} g \bar{\psi} \gamma_{5} \psi\left(\partial_{\mu} X^{\mu}\right)\right],
\end{aligned}
$$

where

$G_{\mu \nu \kappa}=\partial_{\mu} B_{\nu \kappa}+\partial_{\nu} B_{\kappa \mu}+\partial_{\kappa} B_{\mu \nu}$,

is the field strength associated with the Kalb-Ramond field. By considering parity transformations in $5 \mathrm{D}$, we can see that both $\phi$ and $\partial_{\mu} X^{\mu}$ behave as pseudo-scalars in 4D. Therefore, the action (43) is absolutely parity-invariant in 4D. The vector field $X_{\mu}$ is the dual of $C_{\mu \nu \kappa}$

$X^{\mu}:=\frac{1}{\sqrt{6}} \epsilon^{\mu \nu \kappa \lambda} C_{\nu \kappa \lambda}$,

and, by using the gauge transformation (4) of $C_{\mu \nu \kappa}$, we obtain

$X^{\mu \prime}=X^{\mu}+\frac{1}{2 \sqrt{6}} \epsilon^{\mu \nu \kappa \lambda} \partial_{\nu} \xi_{\kappa \lambda}$, and hence

$\partial_{\mu} X^{\mu \prime}=\partial_{\mu} X^{\mu}$

i.e., the vector field $X^{\mu}$ is purely longitudinal. By using the field equations (12) and (13), this dimensional reduction shows that the bosonic fields in the reduced action (43) acquire a mass $m^{2}$. The field $\widetilde{H}^{\bar{\mu}}=\left(\widetilde{G}^{\mu}, \widetilde{H}^{4}\right)$ may be split in $\widetilde{H}^{4}=\frac{1}{\sqrt{6}} \partial_{\mu} X^{\mu}$ and $\widetilde{G}^{\mu}$, i.e., the dual of $G^{\mu \nu \kappa}$ : $\widetilde{G}^{\mu}=(\chi, \vec{Y})$. The dual of $G^{\mu \nu \kappa}$ is given by

$\widetilde{G}_{\mu}=\frac{1}{6} \epsilon_{\mu \nu \kappa \lambda} G^{\nu \kappa \lambda}$.

Therefore, the covariant derivative of (43) in 4 dimensions is

$D_{\mu}=\partial_{\mu}+i e A_{\mu}+i g \widetilde{G}_{\mu}$.

Here, the 3-form gauge field in 4D is nothing but a longitudinal vector, because it propagates its longitudinal part and suppresses its transverse component, as Eq. (47) suggests. The light-shining-through-a-wall experiments (LSW) [47,48] are capable of detecting longitudinal radiation [49].

In connection with the works by Antoniadis et al. [50,51] and Ringwald et al. [49], what they consider in 4D as a pseudo-scalar (axionic electrodynamics), turns out to originate, in our case, from the mixing between the 3-form $\left(X^{\mu}\right)$ and the $\phi \equiv A^{4}$ (pseudo-scalar). So, the Antoniadis' axion is for us a remnant of the 5-dimensional fields in the form of this mixing.

Actually, the papers by Antoniadis [50,51] show that our 3 -form which appears in 4D must in fact be a pseudo-scalar. Our vector field, $X^{\mu}$, just propagates the longitudinal part because this is its gauge-invariant component, i.e., this vector field carries the spin- $\underline{0}$ and the spin- $\underline{1}$ components, but the gauge symmetry (46) acts to gauge away precisely the spin1 piece.

These two new bosons (vector and scalar) that appear simultaneously in our model can be interpreted, in fact, as "two sides of the same coin". A "coin" that is conceived in a 5-dimensional scenario, but, from the point of view of our 4-dimensional world, leads us to see it as if there were two separate entities. However, from the point of view of the 5 -dimensional bulk, it is only one entity, since the 5 dimensions provide a unified view of these two fields. In 4D, we see two entities, the vector and scalar bosons, as a result of dimensional reduction. Under this unified interpretation, the masses of the "two particles" being the same would also suggest that there is a common entity the propagates in the bulk between the branes. Further on, in Sect. 3.3, we shall discuss the split of this mass degeneracy.

\subsection{Considering the gravitational sector}

An issue to be investigated concerns the introduction of the gravitational coupling in the action (36) to subsequently per- 
form a dimensional reduction to 4D. For this purpose, we consider the action (36), now in the presence of gravity, to be given by

$$
\begin{aligned}
S_{5 D}= & \int \mathrm{d}^{5} x \sqrt{-g}\left[-\frac{R}{2 \kappa^{2}}+\bar{\psi}\left(i \gamma^{\bar{\mu}} \mathcal{D}_{\bar{\mu}}-m_{f}\right) \psi\right. \\
& -\frac{1}{4} g^{\bar{\mu} \bar{\alpha}} g^{\bar{v} \bar{\beta}} F_{\bar{\mu} \bar{\nu}} F_{\bar{\alpha} \bar{\beta}}-\frac{1}{8} g^{\bar{\mu} \bar{\alpha}} g^{\bar{\nu} \bar{\beta}} g^{\bar{\rho} \bar{\gamma}} g^{\bar{\lambda} \bar{\sigma}} \\
& \left.\times H_{\bar{\mu} \bar{\nu} \bar{\kappa} \bar{\lambda}} H_{\bar{\alpha} \bar{\beta} \bar{\gamma} \bar{\sigma}}+\frac{m}{\sqrt{6}} \frac{\epsilon^{\bar{\mu} \bar{\nu} \bar{\kappa} \bar{\lambda}} \bar{\rho}}{\sqrt{-g}} A_{\bar{\mu}} \partial_{\bar{\nu}} C_{\bar{\kappa} \bar{\lambda} \bar{\rho}}\right],
\end{aligned}
$$

where $\kappa$ is the gravitational coupling (related to the Newton constant by $\left.\kappa^{2}=8 \pi G\right), R$ is the Ricci scalar, and the covariant derivative, $\mathcal{D}_{\bar{\mu}}$, acting on the fermions contains the spin connection, $\Omega_{\bar{\mu}}$, as given below

$\mathcal{D}_{\bar{\mu}}=\partial_{\bar{\mu}}+i e A_{\bar{\mu}}+i g \widetilde{H}_{\bar{\mu}}+i g^{\prime} \Omega_{\bar{\mu}}$.

The coupling of gravity to fermions requires the vielbein formalism, the so-called first-order approach. Here, we carry out a natural extension of the formalism to 5 dimensions. It is well known in the literature that the metric of the curved space-time is written as $e_{a}^{\bar{\mu}} e_{b}^{\bar{\nu}} g_{\bar{\mu} \bar{\nu}}=\eta_{a b}$, in our case, $\eta^{a b}(+,-,-,-,-)$ is the Minkowski metric on the tangent space, and $e_{a}^{\frac{1}{\mu}}$ is the 5-bein. The spin connection is expanded in the basis of the Lorentz group generators, $\Sigma^{a b}=\frac{i}{4}\left[\gamma^{a}, \gamma^{b}\right]$, as $\Omega_{\bar{\mu}}(x)=\frac{1}{2} \Sigma^{a b} \omega_{\bar{\mu}}^{a b}(x)$, where $a, b=$ $\{0,1,2,3,4\}$ are the frame indices of the Lorentz group. The gamma-matrices, $\gamma^{\bar{\mu}}$, are defined as $\gamma^{\bar{\mu}}=\gamma^{a} e^{\bar{\mu}}{ }_{a}$ and fulfill the Clifford algebra $\left\{\gamma^{\bar{\mu}}, \gamma^{\bar{\nu}}\right\}=e_{a}^{\bar{\mu}} e_{b}^{\bar{v}}\left\{\gamma^{a}, \gamma^{b}\right\}=2 g^{\bar{\mu} \bar{\nu}}$. The components of the spin connection are related to the vielbein and metric as follows:

$$
\begin{aligned}
\omega_{\bar{\mu}}^{a b}= & \frac{1}{2} e_{\bar{\nu}}^{a} \partial_{\bar{\mu}} e^{b \bar{\nu}}+\frac{1}{2} e^{a \bar{\nu}} e^{b \bar{\sigma}} \partial_{\bar{\sigma}} g_{\bar{\mu} \bar{\nu}}-\frac{1}{2} e_{\bar{\nu}}^{b} \partial_{\bar{\mu}} e^{a \bar{\nu}} \\
& -\frac{1}{2} e^{b \bar{\nu}} e^{a \bar{\sigma}} \partial_{\bar{\sigma}} g_{\bar{\mu} \bar{\nu}} .
\end{aligned}
$$

In the sector of the gauge fields, the tensor $F_{\bar{\mu} \bar{\nu}}$ remains unaltered when coupled to the covariant derivative of the curved space-time. The same is true for the 3 -form $H_{\bar{\mu} \bar{\nu} \bar{\rho} \bar{\lambda}}$. The 2- and 3-forms $F_{\bar{\mu} \bar{\nu}}$ and $H_{\bar{\mu} \bar{\nu} \bar{\rho} \bar{\lambda}}$, even if defined with the usual derivatives, behave like tensors and so there is no need to redefine them by replacing the ordinary by the covariant derivatives. Moreover, if the latter are used to redefine $F$ and $G$, the gauge symmetries for $A_{\bar{\mu}}$ and $C_{\bar{\mu} \bar{\nu} \bar{\rho}}$ would be explicitly broken if torsion is present. This is the case, since we have fermions. So, to keep the gauge symmetries, the expressions for $F$ and $H$ are not changed in presence of gravity, and covariance under general coordinate transformations is also guaranteed. To get information on the excitation spectrum of the gravity sector, we take the linear approximation for the gravitational field:

$g_{\bar{\mu} \bar{\nu}}(x)=\eta_{\bar{\mu} \bar{\nu}}+\kappa h_{\bar{\mu} \bar{\nu}}(x)$, where we consider just linear terms in the $\kappa$ constant. In so doing, we obtain the action (50) linearized in $5 \mathrm{D}$ as

$$
\begin{aligned}
S_{5 D}= & \int \mathrm{d}^{5} x\left[-\frac{1}{4}\left(\partial_{\bar{\mu}} h_{\bar{\nu} \bar{\rho}}\right)^{2}+\frac{1}{2}\left(\partial_{\bar{\mu}} h^{\bar{\mu} \bar{\nu}}\right)^{2}+\frac{1}{2} \bar{h} \partial_{\bar{\mu}} \partial_{\bar{\nu}} h^{\bar{\mu} \bar{\nu}}\right. \\
& +\frac{1}{4}\left(\partial_{\bar{\mu}} \bar{h}\right)^{2}++\bar{\psi}\left(i \gamma^{\bar{\mu}} D_{\bar{\mu}}-m_{f}\right) \psi-\frac{1}{4} F_{\bar{\mu} \bar{\nu}}^{2} \\
& \left.-\frac{1}{8} H_{\bar{\mu} \bar{\nu} \bar{\kappa} \bar{\lambda}}^{2}+\frac{m}{\sqrt{6}} \epsilon^{\bar{\mu} \bar{\nu} \bar{\kappa} \bar{\rho} \bar{\rho}} A_{\bar{\mu}} \partial_{\bar{\nu}} C_{\bar{\kappa} \bar{\lambda} \bar{\rho}}+\mathcal{O}(\kappa)\right],
\end{aligned}
$$

where $\bar{h}:=h_{\bar{\mu}}{ }^{\bar{\mu}}$ and we have omitted the terms of order $\mathcal{O}(\kappa)$, which include the gravitational interactions of the fermions and gauge fields, since they are not important for what we shall discuss in the sequel. Actually, we wish to keep track of the interference, in 4D, between the degrees of freedom stemming from the gravitational sector and the bosonic fields in the gauge sector. This is why we include the gravity-fermion interactions in the $\mathcal{O}(\kappa)$-term of the action above. In this action, the $h$-Lagrangian is invariant under the gauge transformation

$h_{\bar{\mu} \bar{\nu}} \longmapsto h_{\bar{\mu} \bar{\nu}}^{\prime}=h_{\bar{\mu} \bar{\nu}}+\kappa^{-1}\left(\partial_{\bar{\mu}} \xi_{\bar{\nu}}+\partial_{\bar{\nu}} \xi_{\bar{\mu}}\right)$,

where $\xi_{\bar{\mu}}$ is any vector function in 5D.

Now, we investigate the dimensional reduction to $4 \mathrm{D}$ in the kinetic terms of the $h$-field by splitting the components $h^{\bar{\mu} \bar{\nu}}=\left\{h^{\mu \nu}, h^{\mu 4}, h^{44}\right\}$, and defining the components $h^{\mu 4}:=V^{\mu}, h^{44}:=\chi$. We adopt the previous condition that $\partial_{4}$ (any field) $=0$, so the $5 \mathrm{D}$ action takes the form below in 4D:

$$
\begin{aligned}
S_{4 D}= & \int \mathrm{d}^{4} x\left[-\frac{1}{4}\left(\partial_{\mu} h_{\nu \rho}\right)^{2}+\frac{1}{2}\left(\partial_{\mu} h^{\mu \nu}\right)^{2}+\frac{1}{2} h \partial_{\mu} \partial_{\nu} h^{\mu \nu}\right. \\
& +\frac{1}{4}\left(\partial_{\mu} h\right)^{2}-\frac{1}{4}\left(\partial_{\mu} V_{\nu}-\partial_{\nu} V_{\mu}\right)^{2}+\bar{\psi}\left(i \gamma^{\mu} D_{\mu}-m_{f}\right) \psi \\
& -\frac{1}{4} F_{\mu \nu}^{2}+\frac{1}{6} G_{\mu \nu \kappa}^{2}-\frac{2 \sqrt{2}}{3} m \epsilon^{\mu \nu \kappa \lambda} A_{\mu} \partial_{\nu} B_{\kappa \lambda} \\
& \left.+\frac{1}{2}\left(\partial_{\mu} \phi\right)^{2}+\frac{1}{2}\left(\partial_{\mu} X^{\mu}\right)^{2}-m \phi \partial_{\mu} X^{\mu}+\mathcal{O}(\kappa)\right], \quad \text { (56) }
\end{aligned}
$$

where $h:=h_{\mu}{ }^{\mu}$. In this expression, we notice the emergence of a new vector field, $V^{\mu}$, and a mixing term of a scalar field, $\chi$, with the weak gravitational field $h^{\mu \nu}$. The kinetic term for the $\chi$-field naturally drops out. It is then reasonable to truncate the $\chi$-field in the reduction, so that $\chi=0$. The kinect term for the vector field $V^{\mu}$ is invariant under the gauge transformation, $V_{\mu} \longmapsto V_{\mu}^{\prime}=V_{\mu}+\kappa^{-1} \partial_{\mu} \xi_{4}(x)$; this is readily checked by making the dimensional reduction in (55). Therefore, we have obtained an action in 4D with three vector fields, $A^{\mu}, V^{\mu}, X^{\mu}$, in which there is no mass term associated to the $V^{\mu}$-field.

3.3 The propagators of the $\left\{A^{\mu}, B^{\nu \kappa}, X^{\alpha}, \phi\right\}$-multiplet

The propagators associated with the Lagrangian (43) are obtained after the inclusion of the corresponding gauge- 
fixing terms:

$$
\begin{aligned}
\mathcal{L}_{g f}=- & \frac{1}{2 \alpha}\left(\partial_{\mu} A^{\mu}\right)^{2}-\frac{1}{2 \beta}\left(\partial_{\mu} B^{\mu \nu}\right)^{2} \\
& -\frac{1}{4 \xi}\left(\partial_{\mu} X_{\nu}-\partial_{\nu} X_{\mu}\right)^{2} .
\end{aligned}
$$

By adding it to the free part of (43), we have $\mathcal{L}_{0}=\mathcal{L}_{04 D}+$ $\mathcal{L}_{g f}$; where

$$
\begin{aligned}
\mathcal{L}_{04 D}= & \bar{\psi}\left(i \gamma^{\mu} D_{\mu}-m_{f}\right) \psi-\frac{1}{4} F_{\mu \nu}^{2}-\frac{1}{2 \alpha}\left(\partial_{\mu} A^{\mu}\right)^{2} \\
& +\frac{1}{6} G_{\mu \nu \kappa}^{2}-\frac{1}{2 \beta}\left(\partial_{\mu} B^{\mu \nu}\right)^{2}-\frac{2 \sqrt{2}}{3} m \epsilon^{\mu \nu \kappa \lambda} A_{\mu} \partial_{\nu} B_{\kappa \lambda} \\
& +\frac{1}{2}\left(\partial_{\mu} X^{\mu}\right)^{2}+\frac{1}{2}\left(\partial_{\mu} \phi\right)^{2}-m \phi \partial_{\mu} X^{\mu} .
\end{aligned}
$$

In the sector of gauge fields, we cast the Lagrangian into the form below:

$$
\begin{aligned}
\mathcal{L}_{04 D}= & \frac{1}{2} A^{\mu} \square\left(\theta_{\mu \nu}+\frac{1}{\alpha} \omega_{\mu \nu}\right) A^{\nu} \\
& -\frac{1}{2} B^{\mu \nu} \square\left[\left(P_{b}^{1}\right)_{\mu \nu, \kappa \lambda}+\frac{1}{2 \beta}\left(P_{e}^{1}\right)_{\mu \nu, \kappa \lambda}\right] B^{\kappa \lambda} \\
& -\frac{1}{2} \phi \square \phi-\frac{1}{2} X^{\mu} \square \omega_{\mu \nu} X^{\nu}-\frac{\sqrt{2}}{3} m A^{\mu} S_{\mu \kappa \lambda} B^{\kappa \lambda} \\
& +\frac{\sqrt{2}}{3} m B^{\kappa \lambda} S_{\kappa \lambda \mu} A^{\mu}-\frac{1}{2} m \phi \partial_{\mu} X^{\mu} \\
& +\frac{1}{2} m X^{\mu} \partial_{\mu} \phi,
\end{aligned}
$$

written in terms of the projection operators:

$$
\begin{aligned}
\theta_{\mu \nu}+\omega_{\mu \nu} & =\eta_{\mu \nu}, \quad \omega_{\mu \nu}=\frac{\partial_{\mu} \partial_{\nu}}{\square} \\
\left(P_{b}^{1}\right)_{\mu \nu, \kappa \lambda} & =\frac{1}{2}\left(\theta_{\mu \kappa} \theta_{\nu \lambda}-\theta_{\mu \lambda} \theta_{\nu \kappa}\right), \\
\left(P_{e}^{1}\right)_{\mu \nu, \kappa \lambda} & =\frac{1}{2}\left(\theta_{\mu \kappa} \omega_{\nu \lambda}-\theta_{\mu \lambda} \omega_{\alpha \kappa}-\theta_{\nu \kappa} \omega_{\mu \lambda}+\theta_{\nu \lambda} \omega_{\mu \kappa}\right),
\end{aligned}
$$

$S_{\mu \nu \kappa}=-m \epsilon_{\mu \nu \kappa \lambda} \partial^{\lambda}$,

which satisfy the relations

$$
\begin{aligned}
& \left(P_{b}^{1}\right)_{\mu \nu, \kappa \lambda}\left(P_{b}^{1}\right)_{, \rho \sigma}^{\kappa \lambda}=\left(P_{b}^{1}\right)_{\mu \nu, \rho \sigma}, \\
& \left(P_{e}^{1}\right)_{\mu \nu, \kappa \lambda}\left(P_{e}^{1}\right)_{, \rho \sigma}^{\kappa \lambda}=\left(P_{e}^{1}\right)_{\mu \nu, \rho \sigma}, \\
& \left(P_{b}^{1}\right)_{\mu \nu, \kappa \lambda}\left(P_{e}^{1}\right)^{\kappa \lambda}, \rho \sigma=0, \\
& \left(P_{e}^{1}\right)_{\mu \nu, \kappa \lambda}\left(P_{b}^{1}\right)_{, \rho \sigma}^{\kappa \lambda}=0 \text {, } \\
& S_{\mu \nu \alpha} S^{\alpha \kappa \lambda}=-2 \square\left(P_{b}^{1}\right)_{\mu \nu}{ }^{, \kappa \lambda}, \\
& \left(P_{b}^{1}\right)_{\mu \nu, \alpha \beta} S^{\alpha \beta \kappa}=S_{\mu \nu}{ }^{\kappa}, \\
& S^{\kappa \alpha \beta}\left(P_{b}^{1}\right)_{\alpha \beta,}^{\mu \nu}=S^{\kappa \mu \nu},
\end{aligned}
$$

$\left(P_{e}^{1}\right)_{\mu v, \alpha \beta} S^{\alpha \beta \kappa}=0$

$\left(P_{e}^{1}\right)_{\mu v, \alpha \beta} S^{\alpha \beta \kappa}=0$,

$S^{\kappa}{ }_{\alpha \beta}\left(P_{e}^{1}\right)^{\alpha \beta, \mu \nu}=0$.

It is convenient to rewrite the Lagrangian in matrix form. For this task, we split the matrix elements as

$P_{\mu \nu}=\square\left(\theta_{\mu \nu}+\frac{1}{\alpha} \omega_{\mu \nu}\right)$,

$Q_{\mu \rho \sigma}=-R_{\mu \nu \sigma}=\frac{2 \sqrt{2}}{3} m S_{\mu \rho \sigma}$,

$\mathbb{S}_{\kappa \lambda, \rho \sigma}=-\square\left[\left(P_{b}^{1}\right)_{\kappa \lambda, \rho \sigma}+\frac{1}{2 \beta}\left(P_{e}^{1}\right)_{\kappa \lambda, \rho \sigma}\right]$.

$W_{\alpha \beta} \equiv-\square \omega_{\alpha \beta}+\frac{1}{\xi} \square \theta_{\alpha \beta}$

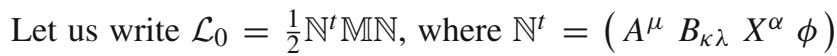
and

$\mathbb{M}=\left(\begin{array}{cccc}P_{\mu \nu} & R_{\mu \rho \sigma} & 0 & 0 \\ Q_{\kappa \lambda \nu} & \mathbb{S}_{\kappa \lambda, \rho \sigma} & 0 & 0 \\ 0 & 0 & W_{\alpha \beta} & m \partial_{\alpha} \\ 0 & 0 & -m \partial_{\beta} & -\square\end{array}\right)$.

After that, we invert the $\mathbb{M}$-matrix to find the propagators listed below:

$$
\begin{aligned}
& \langle\phi \phi\rangle=\frac{i}{k^{2}-m^{2}}, \\
& \left\langle X_{\mu} X_{\nu}\right\rangle=\frac{i}{k^{2}-m^{2}} \frac{k_{\mu} k_{v}}{k^{2}}-i \frac{\xi}{k^{2}}\left(\eta_{\mu \nu}-\frac{k_{\mu} k_{v}}{k^{2}}\right), \\
& \left\langle\phi X^{\mu}\right\rangle=-\left\langle X^{\mu} \phi\right\rangle=\frac{m}{k^{2}-m^{2}} \frac{k^{\mu}}{k^{2}}, \\
& \left\langle A_{\mu} B_{v \kappa}\right\rangle=-\left\langle B_{\mu \nu} A_{\kappa}\right\rangle=\frac{m}{k^{2}-m^{2}} \frac{\epsilon_{\mu \nu \kappa \lambda} k^{\lambda}}{k^{2}}, \\
& \left\langle A_{\mu} A_{\nu}\right\rangle=-\frac{i}{k^{2}-m^{2}}\left[\eta_{\mu \nu}+(\alpha-1) \frac{k_{\mu} k_{\nu}}{k^{2}}\right] \\
& +\alpha \frac{i m^{2}}{k^{2}-m^{2}} \frac{k_{\mu} k_{v}}{\left(k^{2}\right)^{2}}, \\
& \left\langle B_{\mu \nu} B_{\kappa \lambda}\right\rangle=\frac{i}{k^{2}-m^{2}}\left[\mathbb{1}_{\mu \nu, \kappa \lambda}+\left(\beta-\frac{1}{2}\right) \mathbb{K}_{\mu \nu, \kappa \lambda}\right] \\
& -\frac{i \beta m^{2}}{k^{2}-m^{2}} \frac{\mathbb{K}_{\mu \nu, \kappa \lambda}}{k^{2}},
\end{aligned}
$$

where $\mathbb{K}_{\mu \nu, \kappa \lambda}:=\eta_{\mu \kappa} \frac{k_{v} k_{\lambda}}{k^{2}}-\eta_{\mu \lambda} \frac{k_{\kappa} k_{\nu}}{k^{2}}-\eta_{\nu \kappa} \frac{k_{\mu} k_{\lambda}}{k^{2}}+\eta_{\nu \lambda} \frac{k_{\mu} k_{\kappa}}{k^{2}}$, and $\mathbb{1}_{\mu \nu, \kappa \lambda}:=\frac{1}{2}\left(\eta_{\mu \kappa} \eta_{\nu \lambda}-\eta_{\mu \lambda} \eta_{\nu \kappa}\right)$.

In $5 \mathrm{D}$, a 1 -form gauge potential carries 3 on-shell degrees of freedom (d.f.); a 3-form gauge field propagates just 1 onshell d.f. Therefore, we have 4 physical degrees of freedom in the sector of gauge bosons. In 4D, consequently we must have these 4 d.f. distributed among the fields we end up with upon dimensional reduction. Considering the propagators of the $\phi$ - and $X^{\mu}$-sectors ( $\phi$ comes from the Maxwell field in 
5D and behaves as a pseudo-scalar in 4D; $X^{\mu}$ comes from the 3-form in $5 \mathrm{D}$ and is the dual of the corresponding 3 form in $4 \mathrm{D}$, so it does not propagate any on-shell d.f.), it becomes clear that the gauge sector in 4D also carries 4 d.f., as it should be. The other 3 d.f. are carried by the mixed $\left\{A^{\mu}, B^{\nu \kappa}\right\}$-system, in such a way that $A^{\mu}$ propagates 2 d.f., whereas $B^{v \kappa}$ carries 1 d.f., due to its gauge symmetry; this then means that these two fields mixed together describe a single massive and neutral spin-1 gauge particle, which we interpret as a sort of paraphoton. Instead of appearing in a mixed $F F$-term [52], our paraphoton is the particle associated to the $\left\{A_{\mu}-B_{\mu \nu}\right\}$-system with a gauge-invariant mass.

Before ending this section, we should clarify two aspects. The first point concerns the massive pseudo-scalar particle described by the $\left\{\phi, X^{\mu}\right\}$-system. The $5 D \rightarrow 4 D$ reduction clearly shows that the $X^{\mu}$-field appears in 4 dimensions only through its divergence. All terms with $X^{\mu}$ in Eq. (43) exhibit a $\partial \cdot X ; X^{\mu}$ never appears otherwise. This means that we are allowed to actually redefine a newfield: $s \equiv \partial_{\mu} X^{\mu}$, which is then an auxiliary field and can therefore be eliminated through its classical field equation:

$s-m \phi+\frac{i}{\sqrt{6}} g \bar{\psi} \gamma_{5} \psi=0$.

Since $s$ is an auxiliary field, it is correct to replace it in the original action (43) through its algebraic equation above, from which we get the canonical Klein-Gordon action $\left(\frac{1}{2} \partial_{\mu} \phi \partial^{\mu} \phi-\frac{1}{2} m^{2} \phi^{2}\right)$, along with a quartic fermionic interaction term, $\left(\bar{\psi} \gamma_{5} \psi\right)^{2}$. This confirms that the $\left\{\phi, X^{\mu}\right\}$ system describes nothing but a massive pseudo-scalar, which we associate to the axion. The $\left\{A^{\mu}, B^{\nu \kappa}\right\}$-system describes the 3 on-shell d.f. of a neutral massive spin-1 particle. It is, however, mass-degenerate with $\phi$. Nevertheless, the 4-dimensional model does not stand by itself. We suggest, but we do not go through that in detail here (it is not our goal) that $A^{\mu}$ may couple to a Higgs sector in such a way that, upon a spontaneous symmetry breaking induced by this Higgs sector, its mass splits from the axion mass.

We take here the Higgs coupling to $A^{\mu}$ as given by the usual photon-paraphoton kinetic mixing $\chi$-parameter [52]. According to the detailed discussion in the paper by Jaeckel and Ringwald [53-55], $\chi$ ranges between $10^{-16}$ and $10^{-4}$, as consideration based on string theory points to. In our case, if, as stated above, the Higgs-paraphoton coupling is given by a $\chi$-parameter in the range $10^{-16}$ to $10^{-12}$, the axionparaphoton mass splitting lies in the sub-eV range (we recall that $\langle$ Higgs $\rangle \sim 246 \mathrm{GeV}$ ), so that the mass degeneracy is lifted. So, in our axion-paraphoton model, the $\chi$-parameter is also present, but it appears in the Higgs-paraphoton coupling, and it is compatible with axion and paraphoton masses both in the sub-eV scale.

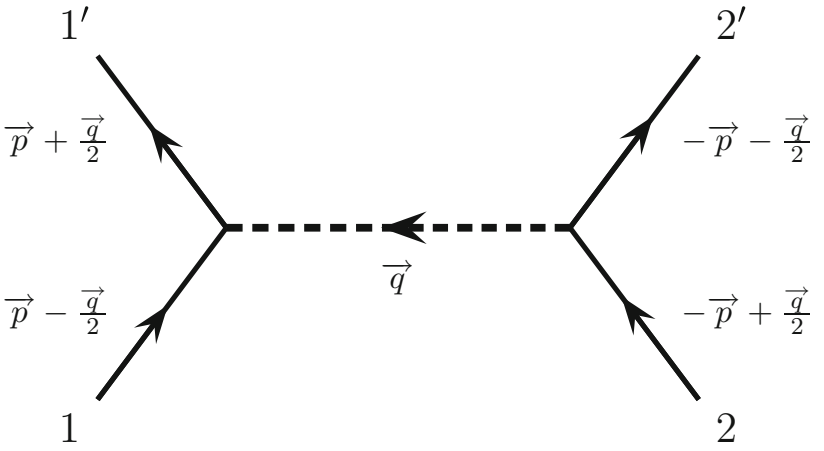

Fig. 1 Momentum assignments in the center-of-mass frame

\subsection{Spin-dependent potentials}

In this section, we study the profiles of the interparticle (nonrelativistic, but spin-and velocity-dependent) potentials when the virtual particles associated to the fields involved in the propagators above are exchanged. These potentials could be suitably extended to macroscopic situations if the exchanged mass, $m$, is small enough. The spin- and velocity-dependent shapes could find some possible application for a physics tested at the sub-millimetric scale, actually, $10^{-1} \mathrm{~mm}$. But in the case considered here, the mass does not break gauge symmetry, so that it would be non-trivial to keep track of the influence of the particular mass mechanism on the form of the interaction particle. We consider the methodology used in $[56,57]$, for which the potential can be obtained, in the first Born approximation, by performing the Fourier integral of the amplitude,

$V(\vec{r}, \vec{v})=-\int \frac{\mathrm{d}^{3} \vec{q}}{(2 \pi)^{3}} e^{i \vec{q} \cdot \vec{r}} \mathcal{A}(\vec{q}, m \vec{v})$.

In the following, we shall use the center-of-mass frame, whose momentum assignments are fixed as in Fig. 1.

We begin by reviewing a well-known case: two pseudoscalar fermionic currents interacting via the scalar propagator $\langle\phi \phi\rangle$, Eq. (79). By applying the Feynman rules, we obtain

$$
\begin{aligned}
i \mathcal{A}^{\langle\phi \phi\rangle}= & \bar{u}\left(p+\frac{q}{2}\right)\left(-e_{1} \gamma_{5}\right) u\left(p-\frac{q}{2}\right)\langle\phi \phi\rangle \\
& \times \bar{u}\left(-p-\frac{q}{2}\right)\left(-e_{2} \gamma_{5}\right) u\left(-p+\frac{q}{2}\right),
\end{aligned}
$$

which can be rewritten in terms of the pseudo-scalar currents as

$\mathcal{A}^{\langle\phi \phi\rangle}=e_{1} e_{2} \frac{J_{1}^{P S} J_{2}^{P S}}{\vec{q}^{2}+m^{2}}$.

So, we take the non-relativistic limit for a pseudo-scalar current (see Eq. (A2) in the appendix) to get

$$
\mathcal{A}^{\langle\phi \phi\rangle}=\frac{e_{1} e_{2}}{4 m_{1} m_{2}} \frac{\left(\vec{q} \cdot\langle\vec{\sigma}\rangle_{1}\right)\left(\vec{q} \cdot\langle\vec{\sigma}\rangle_{2}\right)}{\vec{q}^{2}+m^{2}} .
$$


Finally, we carry out the Fourier integral and obtain [56]:

$V_{P S-P S}^{\langle\phi \phi\rangle}=-\frac{e_{1} e_{2}}{4 m_{1} m_{2}} \mathcal{V}^{\langle\phi \phi\rangle}$,

where we define

$$
\begin{aligned}
\mathcal{V}^{\langle\phi \phi\rangle} & =\left[(1+m r)\left(\langle\vec{\sigma}\rangle_{1} \cdot\langle\vec{\sigma}\rangle_{2}\right)\right. \\
& \left.-\left(3+3 m r+m^{2} r^{2}\right)\left(\hat{r} \cdot\langle\vec{\sigma}\rangle_{1}\right)\left(\hat{r} \cdot\langle\vec{\sigma}\rangle_{2}\right)\right] \frac{e^{-m r}}{4 \pi r^{3}} .
\end{aligned}
$$

Let us now move on to the next case, where we take the $\langle A B\rangle$-propagator of Eq. (82). The amplitude is given by

$$
\begin{aligned}
i \mathcal{A}^{\langle A B\rangle}= & \bar{u}\left(p+\frac{q}{2}\right)\left(-i e_{1} \gamma^{\mu}\right) u\left(p-\frac{q}{2}\right)\left\langle A_{\mu} B_{\kappa \lambda}\right\rangle \\
& \times \bar{u}\left(-p-\frac{q}{2}\right)\left(\frac{g_{2}}{2} \gamma_{\rho} \epsilon^{\rho \nu \kappa \lambda} q_{\nu}\right) u\left(-p+\frac{q}{2}\right) .
\end{aligned}
$$

After some algebraic manipulations, it can be rewritten in terms of vector currents:

$\mathcal{A}^{\langle A B\rangle}=-\frac{e_{1} g_{2} m}{\vec{q}^{2}+m^{2}}\left(J_{1}^{V}\right)^{\mu}\left(J_{2}^{V}\right)_{\mu}$.

If we take the contraction between these currents, Eq. (A6), and perform the Fourier integral, we obtain

$$
V^{\langle A B\rangle}=e_{1} g_{2} m \delta_{1} \delta_{2} \frac{e^{-m r}}{4 \pi r}+e_{1} g_{2} m \mathcal{V}_{(2)}^{\langle A B\rangle},
$$

where we have defined

$$
\begin{aligned}
& \mathcal{V}_{(2)}^{\langle A B\rangle}=\delta_{1} \delta_{2}\left[\left(\frac{1}{m_{1}^{2}}+\frac{1}{m_{2}^{2}}\right)\left(\frac{\vec{p}^{2}}{4}+\frac{m^{2}}{16}\right)+\frac{\vec{p}^{2}}{m_{1} m_{2}}\right] \\
& \frac{e^{-m r}}{4 \pi r}-\left\{\vec{p} \times\left[\frac{1}{4}\left(\frac{\delta_{1}}{m_{2}^{2}}\langle\vec{\sigma}\rangle_{2}+\frac{\delta_{2}}{m_{1}^{2}}\langle\vec{\sigma}\rangle_{1}\right)\right.\right. \\
& \left.\left.+\frac{1}{2} \frac{\left(\delta_{1}\langle\vec{\sigma}\rangle_{2}+\delta_{2}\langle\vec{\sigma}\rangle_{1}\right)}{m_{1} m_{2}}\right]\right\} \cdot \hat{r}(1+m r) \frac{e^{-m r}}{4 \pi r^{2}} \\
& +\left\{\frac{\left(\langle\vec{\sigma}\rangle_{1} \cdot\langle\vec{\sigma}\rangle_{2}\right)}{4 m_{1} m_{2}}\left[1+m r+m^{2} r^{2}\right]\right. \\
& \left.-\frac{\left(\langle\vec{\sigma}\rangle_{1} \cdot \hat{r}\right)\left(\langle\vec{\sigma}\rangle_{2} \cdot \hat{r}\right)}{4 m_{1} m_{2}}\left[3+3 m r+m^{2} r^{2}\right]\right\} \frac{e^{-m r}}{4 \pi r^{3}} .
\end{aligned}
$$

$\delta_{1}$ and $\delta_{2}$ (as explained in detail in the appendix) vanish if particle 1 or particle 2 experience a spin flip in the interaction.

Thus, we notice that the first term in Eq. (94) behaves like a Yukawa term, while $\mathcal{V}_{(2)}^{\langle A B\rangle}$ is suppressed by a factor of $\mathcal{O}\left(v^{2} / c^{2}\right)$.

Finally, we calculate the most involved potential, the one associated with the $\langle B B\rangle$-propagator of Eq. (84). The amplitude assumes the form

$$
\begin{aligned}
i \mathcal{A}^{\langle B B\rangle}= & \bar{u}\left(p+\frac{q}{2}\right)\left(-\frac{g_{1}}{2} \gamma^{\rho} \epsilon_{\rho \xi \mu \nu} q^{\xi}\right) u\left(p-\frac{q}{2}\right)\left\langle B^{\mu \nu} B_{\kappa \lambda}\right\rangle \\
& \times \bar{u}\left(-p-\frac{q}{2}\right)\left(\frac{g_{2}}{2} \gamma_{\alpha} \epsilon^{\alpha \beta \kappa \lambda} q_{\beta}\right) u\left(-p+\frac{q}{2}\right) \\
= & -\frac{g_{1} g_{2}}{4} \epsilon_{\rho \xi \mu \nu} \epsilon^{\alpha \beta \kappa \lambda} q^{\xi} q_{\beta}\left(J_{1}^{V}\right)^{\rho}\left(J_{2}^{V}\right)_{\alpha}\left\langle B^{\mu \nu} B_{\kappa \lambda}\right\rangle .
\end{aligned}
$$

After expressing the product $\epsilon_{\rho \xi \mu \nu} \epsilon^{\alpha \beta \kappa \lambda}$ in terms of Kronecker deltas, this amplitude simplifies as follows:

$$
\begin{aligned}
& i \mathcal{A}^{\langle B B\rangle}=\frac{g_{1} g_{2}}{4}\left\{( J _ { 1 } ^ { V } ) ^ { \mu } ( J _ { 2 } ^ { V } ) _ { \mu } \left[2 q^{2}\left\langle B^{\kappa \lambda} B_{\kappa \lambda}\right\rangle\right.\right. \\
& \left.\quad+4 q_{\beta}\left\langle B^{\beta \kappa} B_{\kappa \lambda}\right\rangle q^{\lambda}\right]+4 q^{2}\left(J_{2}^{V}\right)_{\alpha}\left\langle B^{\alpha \lambda} B_{\lambda \kappa}\right\rangle\left(J_{1}^{V}\right)^{\kappa} \\
& \left.\quad+4\left(J_{2}^{V}\right)_{\alpha} q_{\beta}\left\langle B^{\alpha \beta} B_{\kappa \lambda}\right\rangle\left(J_{2}^{V}\right)^{\kappa} q^{\lambda}\right\} .
\end{aligned}
$$

Its possible to show, after some lengthy evaluations, that the terms associated with the operator $\mathbb{K}$, Eq. (84), do not contribute to the amplitude. Therefore, the amplitude does not depend on the gauge-fixing terms. Then we could take

$\left\langle B_{\mu \nu} B_{\lambda \kappa}\right\rangle=\frac{i}{q^{2}-m^{2}} \mathbb{1}_{\mu \nu, \lambda \kappa}$,

which leads to the following result:

$\mathcal{A}^{\langle B B\rangle}=\frac{g_{1} g_{2}}{2} \frac{\vec{q}^{2}}{\vec{q}^{2}+m^{2}}\left(J_{1}^{V}\right)^{\mu}\left(J_{2}^{V}\right)_{\mu}$.

Once again, we use the contraction (A6). The Fourier integral yields the result:

$V^{\langle B B\rangle}=\frac{g_{1} g_{2} m^{2}}{4} \delta_{1} \delta_{2} \frac{e^{-m r}}{4 \pi r}+\frac{g_{1} g_{2} m^{2}}{4} \mathcal{V}_{(2)}^{\langle A B\rangle}$.

This potential has the same functional form and behavior as the one obtained in the $\langle A B\rangle$-case, Eq. (94).

Now, we shall clarify some points. We emphasize that, by adopting the Scherk-Schwarz dimension reduction scheme, such that we decompose $\bar{\mu}=(\mu, 4)$ and assume $\partial_{4}$ (any field) $=0$, we neglect the non-zero Kaluza-Klein modes and, consequently, the higher-dimensional Planck mass does not play any role in our approach. Thus, the 4-dimensional physics should receive only information on the radius of the extra dimension. This parameter has not explicitly appeared in the potentials simply because we have used the same notation for the couplings constants in 5D and 4D. By carrying out a dimensional analysis of the fields present in the actions $S_{5 D}$ and $S_{4 D}$, we could conclude that, for example, $g^{5 D} / \sqrt{L}=g^{4 D}$, where $L=\int \mathrm{d} x_{4}$ stands for the length of the extra dimension (the $x_{4}$-coordinate). In order to impose constraints on the radius of extra dimension, we should notice that the coupling $g$ always appears together with the mass $m$ in the potentials. So, we need to combine different experiments involving these potentials. We are not following this path here, since this is not within the scope of the present paper. But we understand that this point should be the object of our attention in a forthcoming work. 
The main inheritances from the 5-dimensional physics appear in the vertex interactions. The spin-dependent terms show up only at $\mathcal{O}\left(v^{2} / c^{2}\right)$, since the dimensional reduction fixes the vertex interactions as a result of what we have in $5 \mathrm{D}$. If we study the free Lagrangian directly in 4D, Eq. (43), we have the freedom to fix the interaction by means of (pseudo)vector or (pseudo-)tensor currents. These results are presented in the work of Ref. [58], with spin-dependent terms in first and second orders in $v / c$.

If we adopt other dimensional reduction schemes, we expect to get different results for the potentials. We also point out a new path: we intend to calculate the interparticle potential directly in 5D and then take the dimensional reduction of the 5D-potential, instead of first reducing from $5 \mathrm{D}$ to $4 \mathrm{D}$ to then compute the potential in $4 \mathrm{D}$. The main reason is that, in 5D-Minkowski space, we have two spins, associated with the $S O(4) \cong S U(2) \times S U(2)$-little group of Poincaré group in $5 \mathrm{D}$, while in $4 \mathrm{D}$ we have only one spin, since $S O(3) \cong S U(2)$.

We have preliminary results taking into account the contribution of this new spin in the interparticle potentials. In some situations, there shall appear new corrections to the monopole-dipole and dipole-dipole potentials, which decrease with a power-law dependence on the radius of the extra dimension, which now play a more fundamental role than the renormalized coupling constant. We expect to report on these results soon.

In 4 dimensions, the vector boson $A^{\mu}$ appears as a massive excitation, so that, rather than a Coulomb-like, we have a Yukawa-type potential. Newton's law is reproduced from the inspection of the graviton sector, for it comes from the linearization of $\sqrt{-g} R$ and no mass parameter appears that endows the gravitation with a mass.

If we are to interpret $A^{\mu}$ as a paraphoton in $4 \mathrm{D}$, then the mass parameter $m$ should be constrained by the experiments that fix an upper bound on the axion mass. The axionparaphoton splitting is taken care of by the $\chi$-parameter, which we propose to govern the Higgs-paraphoton coupling.

\section{Concluding comments}

One proposes here to investigate a 5D electromagnetic model with a (Abelian) topological mass term built up in terms of a 1-form and a 3-form gauge potential. Such a description may offer some hint for modeling the so-called dark energy, due to the presence of the $\Theta^{4}{ }_{4}$-component of our energymomentum tensor that may correspond to a negative pressure and may then be describing an expanding system.

Going over into 4D, by following the particular dimensional reduction procedure we have adopted, we identify the emergence of a sector we refer to as an extra dark sector.
It is associated to an excitation that acts as a scalar photon, to which a scalar magnetic-like field is related. In this scenario, in 4D, there emerges a neutral massive vector boson (mass $m$ ) along with a neutral pseudo-scalar with the same mass. The 3-form that yields a negative contribution to the pressure is responsible (with its mixing to the 4D Abelian gauge boson inherited from the 5-dimensional 1-form) for the appearance of two bosons: a longitudinal vector, that is, an auxiliary field, and a massive spin-1 particle, which we interpret as a paraphoton. In our formulation, the axion (the pseudo-scalar component identified as $A^{4}$ ) and the paraphoton turn out to be mass-degenerate, both considered in the sub-eV scale. We propose to couple the paraphoton to the electroweak Higgs scalar with the $\chi$-parameter as the Higgs gauge coupling, so that the axion and the paraphoton have their degeneracy lifted with a splitting also in the sub-eV scale. On the other hand, the massive scalar may be interpreted as the axion remnant of the Electrodynamics in 5D considering that the Chern-Simons term (Abelian) in 5D is defined as $\epsilon^{\bar{\mu} \nu \bar{\lambda} \bar{\kappa} \bar{\mu} \bar{\rho}} A_{\bar{\nu}} F_{\bar{\lambda} \bar{\kappa}} F_{\bar{\mu} \bar{\rho}}$ and its dimensional reduction to 4D leads to the axionic term type: $\theta F_{\mu \nu} \widetilde{F}^{\mu \nu}$ where $A^{4}=\theta$ [47].

By setting $g=0$, i.e., by eliminating the non-minimal coupling described by $\widetilde{G}^{\mu}$ in the covariant derivative, the field $X^{\mu}$ decouples from the fermions; however, the axionic-like particle remains coupled, for its coupling is electromagnetic. We then point out that it is possible to decouple the field $X^{\mu}$, and, at the same time, to keep the axion coupled with the charged fermionic matter. We believe that it would be interesting to consider, from the onset, a Chern-Simons term in 5 dimensions which would naturally induce the axionic coupling in 4D: $\theta F_{\mu \nu} \widetilde{F}^{\mu \nu}$. The 5D Abelian Chern-Simons term is cubic in the gauge field and may provide a very natural scenario to discuss photon self-interactions and non-linear effects, with potentially interesting consequences for the electromagnetic interaction in 4 dimensions. We shall concentrate some efforts on this particular issue and we intend to report on that in a forthcoming paper.

As a final open question, we highlight the study of magnetic monopoles in a 5-dimensional scenario, where they become extended 1-dimensional objects (i.e., strings) that appear as the dual of point-like charges. So, in 5D, magnetic monopoles have their interaction mediated by the 2form Kalb-Ramond field. As a follow-up of the present work, we shall be concentrating efforts to pursue an investigation of $5 \mathrm{D}$ electrodynamics in the presence of (extended) magnetic monopoles, so that a 1-, a 2- and a 3-form should all be present and their effect in connection with negative pressure and the phenomenon of dark energy in 4 dimensions should be reassessed.

Acknowledgments J. Jaeckel and P.C. Malta are acknowledged for fruitful exchange of ideas and for pointing out relevant references. The 
authors express their gratitude to the agencies "Conselho Nacional de Desenvolvimento Científico e Tecnológico" (CNPq-Brazil) and "Fundação Carlos Chagas Filho de Amparo à Pesquisa do Estado do Rio de Janeiro" (FAPERJ) for the financial support.

Open Access This article is distributed under the terms of the Creative Commons Attribution 4.0 International License (http://creativecomm ons.org/licenses/by/4.0/), which permits unrestricted use, distribution, and reproduction in any medium, provided you give appropriate credit to the original author(s) and the source, provide a link to the Creative Commons license, and indicate if changes were made.

Funded by SCOAP ${ }^{3}$.

\section{Appendix A: Currents in the non-relativistic limit}

In this appendix, we collect the currents and their contractions. We consider the same conventions and notations as in Ref. [58]. In the non-relativistic limit, the solution to the Dirac equation, with positive energy, is given by [59]

$u(p) \approx \xi\left(\begin{array}{c}1 \\ \frac{\vec{\sigma} \cdot \vec{p}}{2 m}\end{array}\right)$.

We take $\xi^{\prime}$ for the Dirac conjugate $\bar{u}(p)$. The pseudo-scalar current (PS), following the parametrization for the first vertex of Fig. 1, can be written as

$J_{1}^{P S}=\bar{u}\left(p+\frac{q}{2}\right) i \gamma_{5} u\left(p-\frac{q}{2}\right)=-\frac{i}{2 m_{1}} \vec{q} \cdot\langle\vec{\sigma}\rangle_{1}$,

where we use $\left\langle\sigma_{i}\right\rangle_{1}:=\xi^{\prime \dagger} \sigma_{i} \xi$ to denote the expectation value of the spin matrix, $\sigma_{i}$, of the particle one.

For the vector current $(V)$,

$\left(J_{1}^{V}\right)^{\mu}:=\bar{u}\left(p+\frac{q}{2}\right) \gamma^{\mu} u\left(p-\frac{q}{2}\right) ;$

the $\mu=0$-component yields

$$
\begin{aligned}
& \bar{u}\left(p+\frac{q}{2}\right) \gamma^{0} u\left(p-\frac{q}{2}\right)=\delta_{1}+\frac{\delta_{1}}{2 m_{1}^{2}}\left(\vec{p}^{2}-\frac{\vec{q}^{2}}{8}\right) \\
& +\frac{i}{4 m_{1}^{2}}(\vec{q} \times \vec{p}) \cdot\langle\vec{\sigma}\rangle_{1}
\end{aligned}
$$

where $\delta_{1}:=\xi^{\prime \dagger} \xi$, with $\delta_{1}=0$ if particle 1 changes the spin orientation; otherwise $\delta_{1}=1$. The same is true for $\delta_{2}$.

For the space component, $\mu=i$, we have

$$
\bar{u}\left(p+\frac{q}{2}\right) \vec{\gamma} u\left(p-\frac{q}{2}\right)=\frac{\vec{p}}{m_{1}} \delta_{1}-\frac{i}{2 m_{1}} \vec{q} \times\langle\vec{\sigma}\rangle_{1} .
$$

The second current, associated with particle 2 or the second vertex of Fig. 1 can be obtained by taking $q \rightarrow-q$, $p \rightarrow-p$ and by exchanging the labels $1 \rightarrow 2$.

Finally, we present the result for the contraction of vector currents, neglecting terms of the order $\mathcal{O}\left(v^{3} / c^{3}\right)$,

$$
\begin{aligned}
& \left(J_{1}^{V}\right)^{\mu}\left(J_{2}^{V}\right)_{\mu} \\
& \approx \delta_{1} \delta_{2}+\delta_{1} \delta_{2}\left[\left(\frac{1}{m_{1}^{2}}+\frac{1}{m_{2}^{2}}\right)\left(\frac{\vec{p}^{2}}{4}-\frac{\vec{q}^{2}}{16}\right)+\frac{\vec{p}^{2}}{m_{1} m_{2}}\right] \\
& +(\vec{q} \times \vec{p}) \cdot\left[\frac{i}{4}\left(\frac{\delta_{1}}{m_{2}^{2}}\langle\vec{\sigma}\rangle_{2}+\frac{\delta_{2}}{m_{1}^{2}}\langle\vec{\sigma}\rangle_{1}\right)\right. \\
& \left.+\frac{i}{2} \frac{1}{m_{1} m_{2}}\left(\delta_{1}\langle\vec{\sigma}\rangle_{2}+\delta_{2}\langle\vec{\sigma}\rangle_{1}\right)\right] \\
& -\frac{1}{4} \frac{1}{m_{1} m_{2}}\left\{\vec{q}^{2}\left(\langle\vec{\sigma}\rangle_{1} \cdot\langle\vec{\sigma}\rangle_{2}\right)-\left(\vec{q} \cdot\langle\vec{\sigma}\rangle_{1}\right)\left(\vec{q} \cdot\langle\vec{\sigma}\rangle_{2}\right)\right\} .
\end{aligned}
$$

\section{References}

1. M.B. Green, Class. Quantum Gravity 16, A77 (1999)

2. J. Maldacena, Int. J. Theor. Phys. 38, 1113 (1999)

3. S. Gubser, I. Klebanov, A. Polyakov, Phys. Lett. B 428, 105 (1998)

4. E. Witten, Adv. Theor. Math. Phys. 2, 253 (1998)

5. O. Aharony, S.S. Gubser, J. Maldacena, H. Ooguri, Y. Oz, Phys. Rep. 323, 183 (2000)

6. N. Arkani-Hamed, S. Dimopoulos, G. Dvali, Phys. Lett. B 429, 263 (1998)

7. M. Tegmark, M.A. Strauss, M.R. Blanton, K. Abazajian, S. Dodelson, H. Sandvik, X. Wang, D.H. Weinberg, I. Zehavi, N.A. Bahcall, F. Hoyle, D. Schlegel, R. Scoccimarro, M.S. Vogeley, A. Berlind, T. Budavari, A. Connolly, D.J. Eisenstein, D. Finkbeiner, J.A. Frieman, J.E. Gunn, L. Hui, B. Jain, D. Johnston, S. Kent, H. Lin, R. Nakajima, R.C. Nichol, J.P. Ostriker, A. Pope, R. Scranton, U.C.V. Seljak, R.K. Sheth, A. Stebbins, A.S. Szalay, I. Szapudi, Y. Xu, J. Annis, J. Brinkmann, S. Burles, F.J. Castander, I. Csabai, J. Loveday, M. Doi, M. Fukugita, B. Gillespie, G. Hennessy, D.W. Hogg, I.C.V. Ivezić, G.R. Knapp, D.Q. Lamb, B.C. Lee, R.H. Lupton, R.H. Mckay, P. Kunszt, J.A. Munn, L. O’Connell, J. Peoples, J.R. Pier, M. Richmond, C. Rockosi, D.P. Schneider, C. Stoughton, D.L. Tucker, D.E. Vanden Berk, B. Yanny, D.G. York, Phys. Rev. D 69, 103501 (2004)

8. P. Mészáros, High-Energy Radiation from Magnetized Neutron Stars (University of Chicago Press, Chicago, 1992)

9. K.S. Stelle, Phys. Rev. D 16, 953 (1977)

10. E. Sezgin, P. van Nieuwenhuizen, Phys. Rev. D 21, 3269 (1980)

11. T.S. Koivisto, N.J. Nunes, Phys. Rev. D 80, 103509 (2009)

12. P.J.E. Peebles, B. Ratra, Rev. Mod. Phys. 75, 559 (2003)

13. J. Blas, J. Lizana, M. Pérez-Victoria, J. High Energy Phys. 2013, 1 (2013)

14. B. Döbrich, K. Daumiller, R. Engel, M. Kowalski, A. Lindner, et al., In: 10th Patras Workshop on Axions, WIMPs and WISPs at CERN (2014). arXiv:1410.0200 [physics.ins-det]

15. D. Kazanas, R.N. Mohapatra, S. Nussinov, V.L. Teplitz, Y. Zhang, Nucl. Phys. B 890, 17 (2015)

16. B.A. Ovrut, D. Waldram, Nucl. Phys. B 506, 236 (1997)

17. N. Hitchin, J. Differ. Geom. 55, 547 (2000). arXiv:math/0010054

18. D. Youm, Phys. Rev. D 63, 045004 (2001)

19. M. Graña, J. Polchinski, Phys. Rev. D 65, 126005 (2002)

20. A.A. Gerasimov, S.L. Shatashvili, J. High Energy Phys. 2004, 074 (2004)

21. A. Aurilia, E. Spallucci, Phys. Rev. D 69, 105005 (2004)

22. G. Dvali (2005). arXiv:hep-th/0507215 [hep-th]

23. C. Bizdadea, E.-M. Cioroianu, S.C. Săraru, Int. J. Mod. Phys. A 21, 6477 (2006) 
24. Y.M.S.S. Pei-Ming Ho, Y. Imamura, J. High Energy Phys. 2008, 014 (2008)

25. E.M. Cioroianu, E. Diaconu, S.-C. Săraru, Fortschritte der Physik 57, 535 (2009)

26. J.B. Jiménez, T.S. Koivisto, A.L. Maroto, D.F. Mota, J. Cosmol. Astropart. Phys. 2009, 029 (2009)

27. T.S. Koivisto, D.F. Mota, C. Pitrou, J. High Energy Phys. 2009, 092 (2009)

28. T.S. Koivisto, N.J. Nunes, Phys. Lett. B 685, 105 (2010)

29. T. Ngampitipan, P. Wongjun, J. Cosmol. Astropart. Phys. 2011, $036(2011)$

30. T.S. Koivisto, N.J. Nunes, Phys. Rev. D 88, 123512 (2013)

31. J. Schmude (2012). arXiv:1201.1621 [hep-th]

32. M.C. Diamantini, G. Guarnaccia, C.A. Trugenberger, J. Phys. A: Math. Theor. 47, 092001 (2014)

33. X.-L. Qi, E. Witten, S.-C. Zhang, Phys. Rev. B 87, 134519 (2013)

34. J. Scherk, J.H. Schwarz, Phys. Lett. B 82, 60 (1979)

35. L.F. Abbott, Acta Phys. Polon. B 13, 5 (1982)

36. H.R. Christiansen, M.S. Cunha, J.A. Helayël-Neto, L.R.U. Manssur, A.L.M.A. Nogueira, Int. J. Mod. Phys. A 14, 147 (1999)

37. C.N. Ferreira, J.A. Helayël-Neto, M.B.D.S.M. Porto, Nucl. Phys. B 620, 181 (2002)

38. B. Holdom, Phys. Lett. B 166, 196 (1986)

39. B. Holdom, Phys. Lett. B 259, 329 (1991)

40. S. Davidson, B. Campbell, D. Bailey, Phys. Rev. D 43, 2314 (1991)

41. S. Davidson, M. Peskin, Phys. Rev. D 49, 2114 (1994)

42. A.G. Riess, P.E. Nugent, R.L. Gilliland, B.P. Schmidt, J. Tonry, M. Dickinson, R.I. Thompson, T. Budavári, S. Casertano, A.S. Evans, A.V. Filippenko, M. Livio, D.B. Sanders, A.E. Shapley, H. Spinrad, C.C. Steidel, D. Stern, J. Surace, S. Veilleux, Astrophys. J. 560, 49 (2001)

43. M.S. Turner, A.G. Riess, Astrophys. J. 569, 18 (2002)

44. S. Perlmutter, G. Aldering, G. Goldhaber, R.A. Knop, P. Nugent, P.G. Castro, S. Deustua, S. Fabbro, A. Goobar, D.E. Groom, I.M. Hook, A.G. Kim, M.Y. Kim, J.C. Lee, N.J. Nunes, R. Pain, C.R. Pennypacker, R. Quimby, C. Lidman, R.S. Ellis, M. Irwin, R.G.M.
Mahon, P. Ruiz-Lapuente, N. Walton, B. Schaefer, B.J. Boyle, A.V. Filippenko, T. Math-eson, A.S. Fruchter, N. Panagia, H.J.M. Newberg, W.J. Couch, T.S.C. Project, Astrophys. J. 517, 565 (1999)

45. A.G. Riess, A.V. Filippenko, P. Challis, A. Clocchiatti, A. Diercks, P.M. Garnavich, R.L. Gilliland, C.J. Hogan, S. Jha, R.P. Kirshner, B. Leibundgut, M.M. Phillips, D. Reiss, B.P. Schmidt, R.A. Schommer, R.C. Smith, J. Spyromilio, C. Stubbs, N.B. Suntzeff, J. Tonry, Astron. J. 116, 1009 (1998)

46. M. Kalb, P. Ramond, Phys. Rev. D 9, 2273 (1974)

47. J. Redondo, A. Ringwald, Contemp. Phys. 52, 211 (2011)

48. M. Betz, F. Caspers, M. Gasior, M. Thumm, In: 8th Patras Workshop on Axions, WIMPs and WISPs, Chicago (2013). arXiv:1309.7373 [physi s.ins-det]

49. P. Arias, J. Jaeckel, J. Redondo, A. Ringwald, Phys. Rev. D 82, 115018 (2010)

50. I. Antoniadis, A. Boyarsky, O. Ruchayskiy (2006). arXiv:hep-ph/0606306

51. I. Antoniadis, A. Boyarsky, O. Ruchayskiy, Nucl. Phys. B 793, 246 (2008)

52. M. Ahlers, H. Gies, J. Jaeckel, J. Redondo, A. Ringwald, Phys. Rev. D 77, 095001 (2008)

53. J. Jaeckel, J. Redondo, A. Ringwald, Phys. Rev. Lett. 101, 131801 (2008)

54. J. Jaeckel, Frascati Phys. Ser. 56, 172 (2012). arXiv:1303.1821 [hep-ph]

55. J. Jaeckel, A. Ringwald, Ann. Rev. Nucl. Part. Sci. 60, 405 (2010). arXiv:1002.0329 [hep-ph]

56. J.E. Moody, F. Wilczek, Phys. Rev. D 30, 130 (1984)

57. B.A. Dobrescu, I. Mocioiu, J. High Energy Phys. 2006, 005 (2006)

58. F.A.G. Ferreira, P.C. Malta, L.P.R. Ospedal, J.A. Helayël-Neto, Eur. Phys. J. C 75, 232 (2015). arXiv:1411.3991 [hep-th]

59. L.H. Ryder, Quantum Field Theory (Cambridge University Press, Cambridge, 1996) 